\title{
Influence du type de canulation iléale sur la digestion et le transit chez le jeune veau ruminant
}

\author{
JP Lallès ${ }^{1}$, C Poncet ${ }^{2}$ \\ 1 INRA, laboratoire du Jeune Ruminant, 65, rue de Saint-Brieuc, 35042 Rennes Cedex; \\ 2 INRA, unité de la Dynamique et de Digestion, 63122 Saint-Genès-Champanelle, France
}

\begin{abstract}
Summary - Effects of ileal cannula type on digestion and transit in the young ruminating calf. Digestion kinetic studies were carried out in young ruminant calves fitted with either simple or reentrant ileal cannulae. The latter had a negative effect on feed intake but the distribution of digestion throughout the rumen, small and large intestines remained similar in both groups. The mean retention time of digesta in the entire tract and stomachs was, however, increased by $15-26 \%$ when re-entrant compared to simple cannulae were used.
\end{abstract}

Les études quantitatives et dynamiques de la digestion à la sortie du rumen et de l'intestin grêle sont effectuées à l'aide de canules simples ou réentrantes (MacRae et Wilson, 1977). Les dernières sont traumatisantes, car elles supposent une transection totale de l'intestin, mais permettent de collecter totalement des digesta. Malgré l'apparition de certaines perturbations de la motricité (Wenham et Wyburn, 1980), elles ne semblent pas modifier profondément les processus digestifs chez le mouton adulte (MacRae et Wilson, 1977). Nous rapportons certains effets du type de canulation iléale sur le transit digestif, observés chez le jeune veau ruminant.

Matériel et méthodes - Onze veaux mâles frisons fistulés aux extrémités de l'intestin grêle ont été sevrés entre 7 et 10 sem d'âge à l'aide de foin $(20 \%)$ et d'aliments concentrés $(80 \%$; pois ou tourteau de soja) distribués respectivement en 2 et 8 repas/j (Lallès et Poncet, 1990). Les veaux étaient munis de canules (diamètres 12-18 mm) iléale simple (lot CIS, 6 veaux) ou réentrante (lot $\mathrm{CIR}, 5$ veaux). L'administration de Cr-EDTA dans le rumen et la distribution de foin, d'aliment concentré et de liquide marqués respectivement par de l'ytterbium 169, du cérium 141 et du PEG, ont été utilisées pour mesurer les flux digestifs et le transit, pendant le sevrage et aux âges de 15-16 et 19-20 sem. Une analyse de variance à 3 facteurs a révélé que l'âge et la source de protéines n'ont généralement pas eu d'influence significative sur les paramètres étudiés (Lallès et Poncet, 1990), contrairement au type de canulation iléale. En l'absence d'interaction significative avec les autres facteurs, les effets du type de canulation sont représentés par les moyennes générales (tableau I).

Résultats et discussion - La quantité moyenne journalière de matière sèche (MS) des aliments solides ingérée a été plus faible chez les veaux du lot CIR que chez ceux du lot CIS $(1,4 \pm 0,6$ vs $1,6 \pm 0,7$ $\mathrm{kgMS} / \mathrm{j}, P<0,05)$. Les niveaux d'ingestion n'ont toutefois pas été différents entre lots ( $53 \pm 18$ vs $56 \pm 17 \mathrm{gMS} / \mathrm{kg}^{0,75} / \mathrm{j}, P \geq 0,05$ ) car les veaux du lot CIR ont présenté une vitesse de croissance plus faible que celle des autres veaux ( $386 \pm 178$ vs $469 \pm 309$ $\mathrm{g} / \mathrm{j}, P \geq 0,05)$, notamment en période postchirurgicale. Les digestibilités fécales apparentes de la matière organique $(0,80 \pm$ 0,02 vs $0,82 \pm 0,03, P<0,05$ ) et de l'azote $(0,72 \pm 0,04$ vs $0,74 \pm 0,05, P \geq 0,05)$ ont été légèrement plus faibles chez les veaux du lot CIS que chez ceux du lot CIR. Cependant, les flux digestifs duodénaux et iléaux de nutriments, rapportés aux quantités ingérées, ainsi que l'efficacité de la synthèse microbienne dans le rumen, n'ont pas été significativement affectés $(P \geq$ $0,05)$ par le type de canulation. Les temps 
de séjour moyen du foin, du concentré et de la phase liquide dans l'ensemble du tube digestif et dans les estomacs ont été supérieurs de 15 à $26 \%$ chez les veaux munis d'une canule réentrante par rapport à ceux munis d'une canule simple (tableau I). Nous avons d'ailleurs observé des obstructions occasionnelles des canules iléales réentrantes, témoins des difficultés d'écoulement des digesta et quelquefois causes d'interruption des mesures en cours. Des effets dépressifs de la canulation iléale réentrante sur la croissance chez le veau préruminant ont été mentionnés par Guilloteau et al (1986). Chez le mouton adulte, Wenham et Wyburn (1980) ont montré, par examen radiologique, que la présence d'une canule iléale réentrante entraîne une résistance au passage aboral des digesta associée à une accumulation de ceux-ci en amont du pontage intestinal. Cependant, avec ce même type d'animal, MacRae et Wilson (1977) n'ont pas observé d'effets de la canulation iléale sur la digestion et le transit.

En conclusion, l'emploi de canules iléales réentrantes plutôt que simples a réduit les quantités d'aliments solides consommées par le jeune veau ruminant, sans conséquence importante sur le bilan quantitatif de la digestion dans les diverses parties du tube digestif. Par contre, le temps de séjour moyen des digesta a été fortement accru dans le tube digestif et les estomacs.
Guilloteau P, Toullec R, Grongnet JF, PatureauMirand P, Prugnaud J, Sauvant D (1986) Br J Nutr 55, 571-592

Lallès JP, Poncet C (1990) Livest Prod Sci 24, 333-345

MacRae JC, Wilson S (1977) Br J Nutr 38, 6571

Wenham G, Wyburn RS (1980) J Agric Sci, Camb 95, 539-546

Tableau I. Influence du type de canulation iléale sur le transit digestif.

$$
\begin{array}{cc}
\text { Canule simple } & \text { Canule réentrante } \\
(n=18) & (n=15)
\end{array}
$$

\begin{tabular}{|c|c|c|}
\hline \multicolumn{3}{|c|}{$\begin{array}{l}\text { Temps de séjour } \\
\text { moyen } \\
\text { dans l'ensemble } \\
\text { du tube digestif (h) }\end{array}$} \\
\hline & $43,0 \pm 8,1$ & $50,2 \pm 8,0$ \\
\hline oncentré & $34,8 \pm 3,6$ & $44,5 \pm 8,7$ \\
\hline & $27,5 \pm 5,6$ & $32,9 \pm 4,8$ \\
\hline
\end{tabular}

Temps de séjour

moyen

dans l'ensemble

des estomacs ( $h$ )

$\begin{array}{lll}\text { Foin }^{*} & 30,3 \pm 6,2 & 35,7 \pm 6,3 \\ \text { Concentré* } & 18,2 \pm 8,4 & 25,0 \pm 4,3 \\ \text { Liquide } & 11,7 \pm 3,3 & 14,9 \pm 3,5\end{array}$

$n$ : nombre d'observations; * effet du type de canulation iléale significatif $(P<0,05)$ 